\title{
Robust Power Allocation and Outage Analysis for Secrecy in Independent Parallel Gaussian Channels
}

\author{
Siddhartha Sarma, Kundan Kandhway and Joy Kuri
}

\begin{abstract}
This letter studies parallel independent Gaussian channels with uncertain eavesdropper channel state information (CSI). Firstly, we evaluate the probability of zero secrecy rate in this system for (i) given instantaneous channel conditions and (ii) a Rayleigh fading scenario. Secondly, when non-zero secrecy is achievable in the low SNR regime, we aim to solve a robust power allocation problem which minimizes the outage probability at a target secrecy rate. We bound the outage probability and obtain a linear fractional program that takes into account the uncertainty in eavesdropper CSI while allocating power on the parallel channels. Problem structure is exploited to solve this optimization problem efficiently. We find the proposed scheme effective for uncertain eavesdropper CSI in comparison with conventional power allocation schemes.
\end{abstract}

\section{INTRODUCTION}

By using inherent random noise in communication channels, physical layer security achieves information theoretically secure communications. Researchers have studied and characterized secrecy capacity for different communication systems and channel scenarios ranging from single antenna single hop [1] to multi-antenna multi-hop systems [2]. Later, to improve secrecy capacity, researchers have proposed schemes like MIMO with artificial noise generation [3], jammer assisted transmission [4], cooperative relaying [5], analog network coding [6] and combined relaying-jamming [7] in the context physical layer security.

However, most of the existing literature on physically secure communications considers perfect knowledge of eavesdroppers' channel state information (CSI) - a far fetched assumption. For real world scenarios, e.g., border surveillance, we can only expect partial eavesdropper CSI (e.g. estimated path loss). Recently a few papers have discussed power allocation to improve secrecy for single channel scenarios when no CSI or partial CSI for the eavesdropper's channel is available, either with the help of a jammer or using beamforming or both [2, 4, 8]. But studies involving (robust) optimal power allocation for parallel Gaussian channels with imperfect eavesdropper CSI have received little attention.

The parallel channels serve as a model for wideband wireless communications, channels with inter-symbol interference, block fading channels and multi-antenna systems. The secrecy capacity of parallel channels was studied in [9] and optimal power allocation for the Gaussian scenario was evaluated in [10]. But none of them addressed the imperfect eavesdropper CSI scenario. In the current article, we propose a robust power allocation scheme which ensures minimum secrecy outage when partial eavesdropper CSI is available.

Robust power allocation has appeared in [11] for relay channels without secrecy, in [4] for MISO systems, and in [12]

The authors are with the Department of Electronic Systems Engineering, Indian Institute of Science, Bangalore, Karnataka - 560012, India (e-mail: \{siddharth, kundan, kuri\}@dese.iisc.ernet.in). for amplify and forward relaying in the context of secrecy. However, these works did not consider parallel Gaussian channels. Our contributions are summarized below.

- Approximate instantaneous complete secrecy outage probability for partial eavesdropper CSI. Closed form expression for average complete secrecy outage for fading channels.

- When non-zero secrecy is possible, optimal power allocation to minimize $\operatorname{Pr}\left(R_{s}<R_{s}^{(0)}\right)$, where $R_{s}^{(0)}$ is the target secrecy rate. The proposed technique for this robust power allocation problem bounds the outage probability and leads to a linear fractional program.

- Computationally efficient technique to solve the formulated linear fractional program by exploiting the problem structure. Comparison of this power allocation technique with several conventional schemes with respect to secrecy outage.

\section{SySTEM MOdEL}

We consider a single transmitter (source)-receiver (destination) pair in presence of an eavesdropper. The source can transmit information to the destination using $N$ parallel channels indexed by $i \in \mathcal{N}=\{1,2, \cdots, N\}$. The eavesdropper is passively listening to the source-destination transmission. The $i$ th channel gains for the source to destination channel and the source to eavesdropper channel are denoted by complex numbers $h_{i}$ and $g_{i}$, respectively. The incomplete CSI for the eavesdropper's channel is modeled as: $g_{i}=\widehat{g}_{i}+\tilde{g}_{i}$ where, $\widehat{g}_{i}$ and $\tilde{g}_{i}$ are the estimated channel gain and the unknown error term, respectively. For $i, j \in \mathcal{N}, \tilde{g}_{i}$ and $\tilde{g}_{j}$ are independent. The error $\tilde{g}_{i}$ is a circularly symmetric Gaussian random variable, i.e., $\tilde{g}_{i} \sim \mathcal{C N}\left(0, \epsilon_{i}^{2}\right), \forall i$. Upon transmitting the vector source signal $\mathbf{x}=\left[x_{1}, x_{2}, \cdots, x_{N}\right]^{T}$, the destination and the eavesdropper receive the following signals:

$$
y_{d, i}=h_{i} x_{i}+z_{d, i} \text { and } y_{e, i}=g_{i} x_{i}+z_{e, i}, \forall i \in \mathcal{N} \text {. }
$$

The noise variables $z_{d, i}$ and $z_{e, i}$ are i.i.d. across the $N$ parallel channels, the channel uses over time, and independent of the source signal. All noise variables are circularly symmetric Gaussian random variables with mean 0 and variance 1 . Also, for practical reasons, we have a common power constraint over the parallel channels, i.e., $\sum_{i=1}^{N} \mathbb{E}\left[x_{i}^{2}\right] \leq P$. This assumption is quite practical when the transmitter has limited power supply; also, excessive power use can interfere with other transmitting nodes in radio range.

For parallel independent Gaussian channels, secrecy capacity is attained when each source signal is distributed according to the Gaussian distribution, i.e., $x_{i} \sim \mathcal{C N}\left(0, P_{i}\right)$. Therefore we can write [9]:

$$
C_{s}=\max _{\mathbf{P} \in \mathcal{P}} \sum_{i=1}^{N}\left[\frac{1}{2} \log \left(1+\left|h_{i}\right|^{2} P_{i}\right)-\frac{1}{2} \log \left(1+\left|g_{i}\right|^{2} P_{i}\right)\right]^{+}
$$


where, $\mathcal{P}:=\left\{\mathbf{P}: \sum_{i=1}^{N} P_{i} \leq P, \quad P_{i} \geq 0, \forall i\right\}, \quad \mathbf{P}=$
$\left[P_{1}, P_{2}, \cdots, P_{N}\right]^{T}$ and $[x]^{+}=\max \{0, x\}$.

III. Complete Secrecy Outage Analysis

In Sec. III-A we evaluate the instantaneous complete outage probability $\operatorname{Pr}\left(C_{s}=0 \mid h_{i}, \widehat{g}_{i}, \forall i\right) \sqrt{1}$ - a consequence of imperfect information about eavesdropper's CSI. Sec. ЏII-B computes the same for fading channels.

\section{A. Complete Secrecy Outage for instantaneous channel gains, $\operatorname{Pr}\left(C_{s}=0 \mid h_{i}, \widehat{g}_{i}, \forall i\right)$}

Complete secrecy outage occurs when the receiver's absolute channel gain is less than the corresponding eavesdropper's absolute channel gain, for all channels, i.e., $\left|h_{i}\right| \leq\left|g_{i}\right|, \forall i \in$ $\mathcal{N}$. This scenario leads to zero secrecy rate irrespective of the power allocated.

$$
\begin{aligned}
\operatorname{Pr}\left(C_{s}=0 \mid h_{i}, \widehat{g}_{i}, \forall i\right) & =\operatorname{Pr}\left(\left|h_{i}\right|<\left|g_{i}\right|, \forall i\right) \\
& =\prod_{i=1}^{N} \operatorname{Pr}\left(\left|h_{i}\right|<\left|\widehat{g}_{i}+\tilde{g}_{i}\right|\right) .
\end{aligned}
$$

The last equality is true because channels are independent. We define random variables $X_{1 i}:=\Re\left(\widehat{g}_{i}+\tilde{g}_{i}\right) \sim$ $\mathcal{N}\left(\Re\left(\widehat{g}_{i}\right), \frac{1}{2} \epsilon_{i}^{2}\right)$ and $X_{2 i}:=\Im\left(\widehat{g}_{i}+\tilde{g}_{i}\right) \sim \mathcal{N}\left(\Im\left(\widehat{g}_{i}\right), \frac{1}{2} \epsilon_{i}^{2}\right)$, where $\Re($.$) and \Im($.$) are real and imaginary parts of a complex$ number, respectively. For each channel, the probability can be calculated in the following manner:

$\operatorname{Pr}\left(\left|h_{i}\right|<\left|\widehat{g}_{i}+\tilde{g}_{i}\right|\right)=\operatorname{Pr}\left(\left|h_{i}\right|^{2}<\left|\widehat{g}_{i}+\tilde{g}_{i}\right|^{2}\right)$ $=\operatorname{Pr}\left(\left|h_{i}\right|^{2}<\left(\frac{2 X_{1 i}^{2}}{\epsilon_{i}^{2}}+\frac{2 X_{2 i}^{2}}{\epsilon_{i}^{2}}\right) \frac{\epsilon_{i}^{2}}{2}\right)=\operatorname{Pr}\left(\left|h_{i}\right|^{2}<\chi_{i}^{2} \frac{\epsilon_{i}^{2}}{2}\right)$.

Here, $\chi_{i}^{2}$ is a non-central chi-square random variable with degrees of freedom (d.o.f) 2 and non-centrality parameter $\lambda_{i}^{2}=2\left(\frac{\Re\left(\widehat{g}_{i}\right)}{\epsilon_{i}}\right)^{2}+2\left(\frac{\Im\left(\widehat{g}_{i}\right)}{\epsilon_{i}}\right)^{2}=\frac{2\left|\widehat{g}_{i}\right|^{2}}{\epsilon_{i}^{2}}$. A non-central chisquare random variable can be approximated by a central chisquare random variable as follows [13]:

$\operatorname{Pr}\left(\chi_{i}^{2}<\eta_{i}\right) \approx \operatorname{Pr}\left(\chi_{i, 0}^{2}<\frac{\eta_{i}}{\left(1+\lambda_{i}^{2} / 2\right)}\right)=1-e^{-\frac{\eta_{i}}{2\left(1+\lambda_{i}^{2} / 2\right)}}$.

For small values of centrality parameter $\left(\lambda_{i}^{2}<0.4\right)$, this approximation is quite accurate and for higher values it is conservative. The last equality is because a central chi-square random variable with d.o.f. 2, is distributed exponentially. Therefore, the final outage probability is

$$
\prod_{i=1}^{N} \operatorname{Pr}\left(\chi_{i}^{2}>\frac{2\left|h_{i}\right|^{2}}{\epsilon_{i}^{2}}\right) \approx \prod_{i=1}^{N} e^{-\frac{\left|h_{i}\right|^{2}}{\epsilon_{i}^{2}\left(1+\lambda_{i}^{2} / 2\right)}}=e^{-\sum_{i=1}^{N} \frac{\left|h_{i}\right|^{2}}{\left|\widehat{g}_{i}\right|^{2}+\epsilon_{i}^{2}}}
$$

In Fig. 11 we plot the outage probability calculated from simulation and analytical approximation with respect to $\epsilon_{i}^{2}$ for several values of $N$ and $\left|\widehat{g}_{i}\right|$. Except for the initial part, where $\lambda_{i}^{2} \nless 0.4$, the approximation is close to the simulation. $B$. Complete secrecy outage for fading channels $\operatorname{Pr}\left(C_{s}=0\right)$

When $h_{i}$ and $\widehat{g}_{i}$ are sampled from circularly symmetric Gaussian distributions $\mathcal{C N}\left(0, \sigma_{m, i}^{2}\right)$ and $\mathcal{C N}\left(0, \sigma_{e, i}^{2}\right)$, respectively, then $\left|h_{i}\right|$ and $\left|g_{i}\right|$ have Rayleigh distributions with parameters $\sigma_{m, i} / \sqrt{2}$ and $\left(\sigma_{e, i}+\epsilon_{i}\right) / \sqrt{2}$. As $\left|h_{i}\right|^{2}$ and $\left|g_{i}\right|^{2}$

\footnotetext{
${ }^{1}$ The precise expression is: $\operatorname{Pr}\left(C_{s}=0 \mid H_{i}=h_{i}, \widehat{G}_{i}=\widehat{g}_{i}, \forall i\right)$. Here, $H_{i}$ and $G_{i}=\widehat{G}_{i}+\tilde{g}_{i}$ are the random variables corresponding to the sourcedestination and the source-eavesdropper channels respectively. The sourcedestination channel gain $H_{i}=h_{i}$ is known perfectly and estimated sourceeavesdropper channel gain is $\widehat{G}_{i}=\widehat{g}_{i}$ at each time epoch. Note that, $\tilde{g}_{i}$ is the uncertainty term and therefore, a random variable. For brevity, we have used the compression $\operatorname{Pr}\left(C_{s}=0 \mid h_{i}, \widehat{g}_{i}\right)$ instead.
}

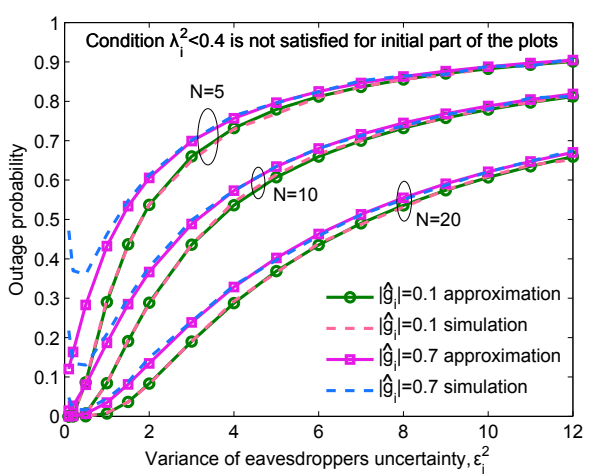

Fig. 1: Plot of outage probability for numerical simulation and approximation with respect of $\epsilon_{i}^{2}$. Here, $\left|h_{i}\right|=0.5, \forall i$.

are exponentially distributed with parameters $1 / \sigma_{m, i}^{2}$ and $1 /\left(\sigma_{e, i}+\epsilon_{i}\right)^{2}$, respectively, following [1]:

$\operatorname{Pr}\left(C_{s}=0\right)=\prod_{i=1}^{N}\left(\frac{1}{1+\rho_{i}}\right)$, where $\rho_{i}=\frac{\sigma_{m, i}^{2}}{\left(\sigma_{e, i}+\epsilon_{i}\right)^{2}}$.

\section{ANALYSIS FOR NON-ZERO SECRECY RATE}

In Sec. III, non-zero secrecy is not possible (irrespective of the power allocation). In contrast, in Sec. IV-A we formulate and provide the optimal solution for a robust power allocation problem to minimize the outage probability for a target secrecy rate, $R_{s}^{(0)}$, i.e., $\operatorname{Pr}\left(R_{s}<R_{s}^{(0)} \mid h_{i}, \widehat{g}_{i}, \forall i\right)$. An expression for main channel outage for a target rate $R_{s}^{(0)}$ is also provided (Sec. IV-B). Only for this section, we use a low SNR approximation of Eq. (1). This is valid for small values of $P$-typical in low power devices, such as, small sensor nodes deployed for surveillance. Several commercial transceivers used in sensor nodes (e.g., ADF7020, ATA542X and CC1000 Series) have linear characteristics for significant portion of SNR [14].

\section{A. Robust optimal power allocation}

Using $\ln (1+x) \approx x$, for $x \rightarrow 0$, we can approximate the secrecy rate in Eq. (1) as:

$$
R_{s}=\frac{1}{2 \ln (2)} \sum_{i=1}^{N}\left[\left|h_{i}\right|^{2}-\left|\widehat{g}_{i}+\tilde{g}_{i}\right|^{2}\right]^{+} P_{i} .
$$

For scenarios when non-zero secrecy is possible, we minimize the outage with respect to a target secrecy rate, $R_{s}^{(0)}$. As eavesdropper's CSI is imperfect, we can not directly maximize $R_{s}$; therefore, we need a robust power allocation approach to minimize the outage probability. The optimization problem can be written as:

$$
\min \operatorname{Pr}\left(R_{s}<R_{s}^{(0)} \mid h_{i}, \widehat{g}_{i}, \forall i\right), \text { subject to: } \mathbf{P} \in \mathcal{P} \text {. }
$$

This optimization is needed only when the main channel, i.e., source to destination channel can sustain a rate $R_{s}^{(0)}$. Otherwise, the secrecy rate is assured to be less than $R_{s}^{(0)}$ and the objective $\operatorname{Pr}\left(R_{s}<R_{s}^{(0)}\right)=1$ everywhere in the feasible set, $\mathbf{P} \in \mathcal{P}$. For small SNR, the main channel can sustain a rate $R_{s}^{(0)}$ when

$$
\sum_{i=1}^{N}\left|h_{i}\right|^{2} P_{i}-2 \ln (2) R_{s}^{(0)}>0 .
$$

Here, the secrecy outage can be calculated from Eq. (2) as: 


$$
\begin{aligned}
& \operatorname{Pr}\left(R_{s}<R_{s}^{(0)} \mid h_{i}, \widehat{g}_{i}, \forall i\right) \\
& \leq \operatorname{Pr}\left(\sum_{i=1}^{N}\left|h_{i}\right|^{2} P_{i}-2 \ln (2) R_{s}^{(0)}<\sum_{i=1}^{N}\left|\widehat{g}_{i}+\tilde{g}_{i}\right|^{2} P_{i}\right) \\
& =\operatorname{Pr}\left(\sum_{i=1}^{N}\left|h_{i}\right|^{2} P_{i}-2 \ln (2) R_{s}^{(0)}<\sum_{i=1}^{N} \chi_{i}^{2} \frac{\epsilon_{i} P_{i}}{2}\right) .
\end{aligned}
$$

As discussed in Sec. ЏI-A $\chi_{i}^{2}$ is a non-central chi-square random variable (d.o.f. 2$)^{2}$. Therefore, the mean of $\chi_{i}^{2} \frac{\epsilon_{i} P_{i}}{2}$ is $\mathbb{E}\left[\chi_{i}^{2} \frac{\epsilon_{i} P_{i}}{2}\right]=\frac{\epsilon_{i} P_{i}}{2}\left(2+\lambda_{i}^{2}\right)=\frac{\epsilon_{i} P_{i}}{2}\left(2+\frac{2\left|\widehat{g}_{i}\right|^{2}}{\epsilon_{i}^{2}}\right)=\left(\epsilon_{i}^{2}+\left|\widehat{g}_{i}\right|^{2}\right) P_{i}$. Using the Markov inequality, we can bound the outage probability as follows:

$$
\begin{aligned}
& \operatorname{Pr}(\sum_{i=1}^{N} \chi_{i}^{2} \frac{\epsilon_{i} P_{i}}{2}>\underbrace{\left.\sum_{i=1}^{N}\left|h_{i}\right|^{2} P_{i}-2 \ln (2) R_{s}^{(0)}\right)}_{\Delta} \\
\leq & \frac{\mathbb{E}\left[\sum_{i=1}^{N} \chi_{i}^{2} \frac{\epsilon_{i} P_{i}}{2}\right]}{\sum_{i=1}^{N}\left|h_{i}\right|^{2} P_{i}-2 \ln (2) R_{s}^{(0)}}=\frac{\sum_{i=1}^{N}\left(\epsilon_{i}^{2}+\left|\widehat{g}_{i}\right|^{2}\right) P_{i}}{\sum_{i=1}^{N}\left|h_{i}\right|^{2} P_{i}-2 \ln (2) R_{s}^{(0)}} .
\end{aligned}
$$

The validity of Markov bound requires $\Delta \geq 0$ in Eq. (7); this is ensured by Eq. (4). The Markov bound is known to be loose; however, we believe that the proposed power allocation scheme has value as substantiated by the numerical results in Sec. V] which show improvement over several conventional schemes. In addition, this approach leads to an easy-to-compute solution (Proposition 1) on resourceconstrained sensor nodes.

To minimize the outage, we propose to minimize this upper bound. This leads to the following equivalent linear fractional program (approximation to Problem (3)):

$$
\min \frac{\mathbf{c}^{T} \mathbf{P}}{\mathbf{d}^{T} \mathbf{P}-2 \ln (2) R_{s}^{(0)}}, \text { subject to: } \mathbf{P} \in \mathcal{P} .
$$

Here, $c_{i}=\epsilon_{i}^{2}+\left|\widehat{g}_{i}\right|^{2}, d_{i}=\left|h_{i}\right|^{2}$, and $\mathbf{c}, \mathbf{d}$ are vectors of these elements. The denominator $\mathbf{d}^{T} \mathbf{P}-2 \ln (2) R_{s}^{(0)} \neq 0$ from the earlier discussion. This linear fractional program can be solved numerically by reformulating it as a linear program using the Charnes-Cooper transformation. However, due to the simplex constraint and only a few variables (the number of parallel channels, $N$ ), we propose the following easy to compute solution 3 .

Proposition 1. The optimal solution to Problem (8) lies in one of the corners of the set $\overline{\mathcal{P}}:=\left\{\mathbf{P}: \sum_{i=1}^{N} P_{i}=P, P_{i} \geq 0, \forall i\right\}$, i.e., $P_{i}=P$ for some $i \in \mathcal{N}$ and $P_{j}^{i=1}=0, \forall j \neq i$.

Proof: We provide an outline of the proof here. One can verify that, at the optimum, the objective function of Problem (2) will consume the total budget $P$ (when at least one of the coefficients of $P_{i}$ is non-zero). Therefore, we can use equality in the sum constraint. From [15], a linear fractional program attains its optimum at the basic feasible solution of

\footnotetext{
${ }^{2}$ We emphasize that non-central to central chi-square approximation is not used/required in this section.

${ }^{3}$ Unlike our case, when the number of corner points is large, simplex or interior-point methods are efficient.
}

the constraint set 4 . The corners of the constraint hyperplaneonly $N$ in number-are the basic feasible solutions and the one that minimizes the objective function is the optimum.

Note that, unlike a general linear (fractional) program where calculating the corner points is computationally costly, in our case, the corners are known and fixed over the parameter set (specified in Proposition 1). Thus, the optimum is computed by enumerating the objective value at each corner point and selecting the best channel-computationally efficient even for a sensor node.

\section{B. Main channel outage for fading scenarios (for small SNR)}

For completeness, we evaluate the probability of the event when the optimization problem (8) need not be solved, as the main channel capacity itself is less than the target rate. The fading coefficients $\left|h_{i}\right| \sim$ Rayleigh $\left(\sigma_{m, i} / \sqrt{2}\right), \forall i$. The outage occurs when the strongest of the $N$ parallel channels cannot sustain the target rate, i.e.,

$$
\begin{aligned}
& \operatorname{Pr}\left(\max _{i}\left\{\left|h_{i}\right|^{2} P\right\}-2 \ln (2) R_{s}^{(0)}<0\right) \\
& =\operatorname{Pr}\left(\bigcap_{i=1}^{N}\left\{\left|h_{i}\right|^{2} P<2 \ln (2) R_{s}^{(0)}\right\}\right) \\
& \stackrel{(u)}{=} \prod_{i=1}^{N}\left(\left|h_{i}\right|^{2} P<2 \ln (2) R_{s}^{(0)}\right) \stackrel{(v)}{=} \prod_{i=1}^{N}\left(1-e^{-\left(\frac{2 \ln (2) R_{s}^{(0)}}{P \sigma_{m, i}^{2}}\right)}\right) .
\end{aligned}
$$

$(u)$ and $(v)$ are true because $h_{i}, \forall i$ are independent and $\left|h_{i}\right|^{2}$ follows the exponential distribution.

\section{Numerical Results}

In this section, we compare several power allocation schemes for parallel independent Gaussian channels and show the effectiveness of our proposed scheme that considers eavesdropper's channel uncertainty (optimization problem (8)). We consider three conventional power allocation strategies for comparison: (a) Equal Power - allocates equal power on every channel, $P_{i}=P / N, \forall i \in \mathcal{N}$. (b) Optimum Capacity power allocation-maximizes the Shannon capacity in the main channel, i.e., $\mathbf{P}_{c}^{*}=\arg \max \sum_{i=1}^{N}\left|h_{i}\right|^{2} P_{i}$ (assuming small SNR), where $\mathbf{P}_{c}^{*} \in \mathcal{P}$. It allocates the power budget $P$ to the strongest main channel. (c) Optimum secrecy power allocation-maximizes secrecy rate for the estimated eavesdropper channel gain without considering uncertainty. $\mathbf{P}_{s}^{*}=\arg \max \sum_{i=1}^{N}\left[\left|h_{i}\right|^{2}-\left|\widehat{g}_{i}\right|^{2}\right]^{+} P_{i}$, where $\mathbf{P}_{s}^{*} \in \mathcal{P}$. It allocates $P$ to the channel that has largest value of $\left|h_{i}\right|^{2}-\left|\widehat{g}_{i}\right|^{2}$.

For simulations, we have generated the main channel $h_{i}$, and the estimated eavesdropper channel $\widehat{g}_{i}$, from $\mathcal{C N}\left(0, \sigma_{m, i}^{2}\right)$ and $\mathcal{C N}\left(0, \sigma_{e, i}^{2}\right)$, respectively with $\sigma_{m, i}^{2}=0.6$ and $\sigma_{e, i}^{2}=0.3$ for all parallel channels. The power budget considered is $P=0.1$. We use the default parameters for the target secrecy rate $R_{s}^{(0)}=0.625 P \times \sigma_{m, i}^{2} / 2 \ln (2)$ and the number of parallel channels $N=10$. Uncertainty in the $i$ th eavesdropper's channel is generated from $\mathcal{C N}\left(0, \epsilon_{i}^{2}\right)$ with $\epsilon_{i}^{2}=0.3$ for $i \in\{1,2, \cdots,\lceil N / 2\rceil\}$ and $\epsilon_{i}^{2}=0.09$ for $i \in\{\lceil N / 2\rceil+1, \cdots, N\}$.

\footnotetext{
${ }^{4}$ This is easy to see because the gradient of the objective function in 8 is non-zero in the constraint set, leading to behavior similar to linear programs.
} 


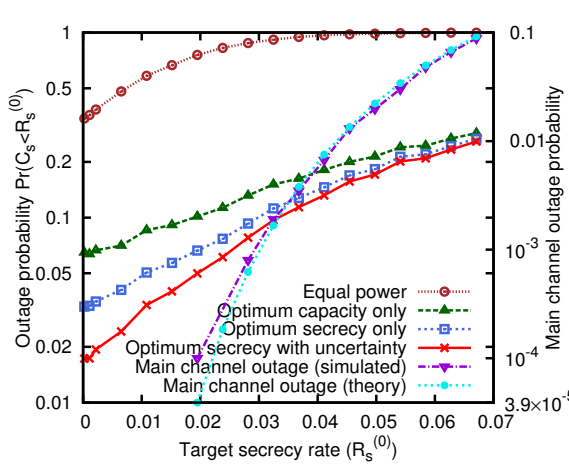

(a) Outage vs. $R_{s}^{(0)}$

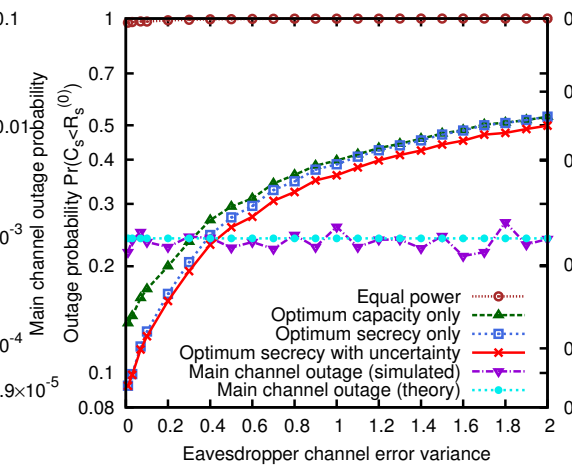

(b) Outage vs. eavesdropper's channel uncertainty

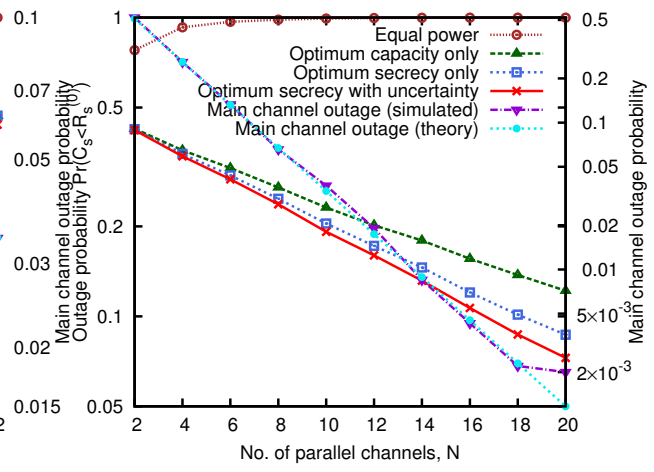

(c) Outage vs. $N$

Fig. 2: Comparison of secrecy outage with respect to different system parameters. Default parameter values (except the one varied): $P=0.1$, $\sigma_{m, i}^{2}=0.6, \sigma_{e, i}^{2}=0.3, R_{s}^{(0)}=0.625 P \times \sigma_{m, i}^{2} / 2 \ln (2), \epsilon_{i}^{2}=0.3$ for $i \in\{1,2, \cdots,\lceil N / 2\rceil\}$ and $\epsilon_{i}^{2}=0.09$ for $i \in\{\lceil N / 2\rceil+1, \cdots, N\}$ and $N=10$.

Fig. 2 plots the variation of the objective function, secrecy outage $\operatorname{Pr}\left(R_{s}<R_{s}^{(0)}\right)$, with respect to variation of the system parameters (left y-axis); and the main channel outage for the same target rate $R_{s}^{(0)}$ (right y-axis). In all the three figures, "Optimum secrecy with uncertainty" identifies the proposed scheme. The plot $\operatorname{Pr}\left(R_{s}<R_{s}^{(0)}\right)$ is calculated, for example, for the proposed scheme as follows.

We generate $h_{i}, \widehat{g}_{i}, i \in \mathcal{N}$. Given this channel, we calculate the power allocation of the proposed scheme $\mathbf{P}_{p}^{*}$ using the optimization problem (8). Note that, we only need the variance of the uncertainty of the eavesdropper's channel, $\epsilon_{i}^{2}, \forall i$ and not the exact value of $g_{i}$ to calculate $\mathbf{P}_{p}^{*}$. Now, the conditional outage probability given $h_{i}$ and $\widehat{g}_{i}, \forall i$, i.e., $\operatorname{Pr}\left(R_{s}<R_{s}^{(0)} \mid h_{i}, \widehat{g}_{i}, \forall i\right)$ is calculated by Monte-Carlo averaging over $10^{4}$ values of $\tilde{g}_{i}, \forall i$ using (6) for $\mathbf{P}_{p}^{*}$. We obtain the final objective function, $\operatorname{Pr}\left(R_{s}<R_{s}^{(0)}\right)$, as follows: $10^{4}$ instances of $h_{i}$ and $\widehat{g}_{i}$ are generated, (6) is averaged over the instances for which the main channel is not in outage, i.e., $\max \left\{\left|h_{i}\right|^{2}, \forall i\right\} P>2 \ln (2) R_{s}^{(0)}$. The same is carried out for the three conventional power allocation strategies.

Fig. 2a demonstrates the effectiveness of the proposed scheme when the target secrecy rate $R_{s}^{(0)}$ is varied, specially for small values of $R_{s}^{(0)}$. The effect of variation of eavesdropper's channel uncertainty is shown in Fig $2 \mathrm{~b}$ As expected, when uncertainty is small, performance of the "Optimum secrecy" power allocation is comparable to the proposed scheme. However, as the uncertainty increases, the proposed scheme outperforms the others. Finally, we vary the number of channels, $N$ in Fig. 2c. The proposed scheme exploits the uncertainty parameter $\epsilon_{i}^{2}$, in addition to the channel gains, and therefore, outperforms "Optimum secrecy" (and others). The improvements are prominent for higher values of $N$.

\section{CONCLUSION}

We study the effect of a single eavesdropper's channel uncertainty in a parallel independent Gaussian channel communications system. We evaluate complete secrecy outage probability for instantaneous and fading channels. Further, we propose a robust power allocation scheme to minimize secrecy outage probability at a target rate in the low SNR regime. Our techniques involve (1) approximating non-central chi-square random variables by corresponding central ones to evaluate instantaneous outage; and (2) bounding outage

probabilities for robust power allocation, which leads to a linear fractional program. Exploiting the structure of the problem, we propose an easy-to-compute solution. Numerical results show the superiority of the proposed scheme compared to the conventional schemes that do not consider uncertainty.

\section{REFERENCES}

[1] J. Barros and M. Rodrigues, "Secrecy capacity of wireless channels," in IEEE International Symposium on Information Theory, July 2006, pp. $356-360$.

[2] D. Ng, E. Lo, and R. Schober, "Secure Resource Allocation and Scheduling for OFDMA Decode-and-Forward Relay Networks," IEEE Trans. on Wireless Commun., vol. 10, no. 10, pp. 3528-40, Oct 2011.

[3] S. Goel and R. Negi, "Guaranteeing secrecy using artificial noise," IEEE Transactions on Wireless Communications, vol. 7, no. 6, pp. 2180-89, Jun 2008.

[4] J. Huang and A. Swindlehurst, "Robust Secure Transmission in MISO Channels Based on Worst-Case Optimization," IEEE Trans. Signal Process., vol. 60, no. 4, pp. 1696-1707, April 2012.

[5] L. Dong, Z. Han, A. Petropulu, and H. Poor, "Improving Wireless Physical Layer Security via Cooperating Relays," IEEE Transactions on Signal Processing, vol. 58, no. 3, pp. 1875 -1888, March 2010.

[6] J. Chen, R. Zhang, L. Song, Z. Han, and B. Jiao, "Joint Relay and Jammer Selection for Secure Two-Way Relay Networks," IEEE Transactions on Information Forensics and Security, vol. 7, no. 1, pp. 310-320, Feb 2012.

[7] K.-H. Park, T. Wang, and M.-S. Alouini, "On the Jamming Power Allocation for Secure Amplify-and-Forward Relaying via Cooperative Jamming," IEEE Journal on Selected Areas in Communications, vol. 31, no. 9, pp. 1741-1750, September 2013.

[8] H.-M. Wang, M. Luo, X.-G. Xia, and Q. Yin, "Joint Cooperative Beamforming and Jamming to Secure AF Relay Systems With Individual Power Constraint and No Eavesdropper's CSI," IEEE Signal Processing Letters, vol. 20, no. 1, pp. 39-42, 2013.

[9] Z. Li, R. Yates, and W. Trappe, "Secrecy Capacity of Independent Parallel Channels," in Securing Wireless Communications at the Physical Layer, R. Liu and W. Trappe, Eds. Springer US, 2010, pp. 1-18.

[10] E. Jorswieck and A. Wolf, "Resource Allocation for the Wire-tap Multicarrier Broadcast Channel," in International Conference on Telecommunications, 2008 (ICT 2008), Jun 2008, pp. 1-6.

[11] S. Mallick, R. Devarajan, M. Rashid, and V. Bhargava, "Resource allocation for selective relaying based cellular wireless system with imperfect csi," IEEE Transactions on Communications, vol. 61, no. 5, pp. 1822-1834, May 2013.

[12] L. Li, Z. Chen, and J. Fang, "Robust transmit design for secure af relay networks based on worst-case optimization," in IEEE International Conference on Acoustics, Speech and Signal Processing (ICASSP), May 2014, pp. 2719-2723.

[13] D. R. Cox and N. Reid, "Approximations to noncentral distributions," Canadian Journal of Statistics, vol. 15, no. 2, pp. 105-114, 1987.

[14] V. Sharma, U. Mukherji, V. Joseph, and S. Gupta, "Optimal energy management policies for energy harvesting sensor nodes," IEEE Trans. Wireless Commun., vol. 9, no. 4, pp. 1326-36, Apr 2010.

[15] K. Swarup, "Letter to the editor-Linear fractional functionals programming," Operations Research, vol. 13, no. 6, pp. 1029-1036, 1965. 